\title{
Molecular Characterization of Strongly and Weakly Interfacially Active Asphaltenes by High-Resolution Mass Spectrometry
}

\author{
Dewi A. Ballard, Martha L. Chacón-Patiño, Peiqi Qiao, Kevin J. Roberts, Robert Rae, Peter J. Dowding, \\ Zhenghe $\mathrm{Xu}$, and David Harbottle*
}

Cite This: Energy Fuels 2020, 34, 13966-13976

Read Online

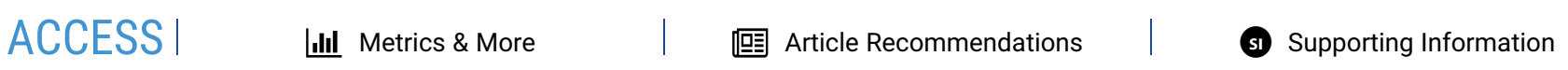

ABSTRACT: Asphaltenes are a complex mixture of molecular structures with a variety of functionalities, which in turn impacts their physical properties. Discriminating between asphaltenes that are strongly and weakly interfacially active is providing a new direction to mitigate asphaltene-related problems. Whole asphaltenes (WA) were extracted from a South American heavy crude oil, further fractionated into interfacially active asphaltenes (IAA) and remaining asphaltenes (RA), and molecularly characterized by positiveion (+) atmospheric pressure photoionization (APPI) using a 9.4 T Fourier transform ion cyclotron mass spectrometer (FT-ICR MS). The IAA fraction was found to contain a greater abundance of heteroatoms with $>50 \%$ of IAA containing two or more heteroatoms as compared to $\sim 30 \%$ for RA. The IAA fraction was enriched in oxygen-containing species, more specifically higherorder $\mathrm{O}_{x}$ and $\mathrm{O}_{x} \mathrm{~S}_{y}$ groups that were predominantly of low DBE. Gas-phase fragmentation of RA and IAA precursor ions $(m / z$ 650) by infrared multiphoton dissociation (IRMPD) revealed an abundance of multi-core motifs in IAA, while RA was found to be a mixture of single-core and multi-core structures. Analysis of the fragmented ions showed a prevalence of nitrogen-containing species of high DBE (aromatic molecular structures), while oxygen-containing species were most likely associated with aliphatic side chains. Extrography fractionation of RA and IAA verified the abundance of multi-core motifs in IAA, which were highly polar and of low DBE and carbon number. These "atypical" structures of IAA are classified as asphaltenes as a result of their functionality and polarity rather than high aromaticity.

\section{INTRODUCTION}

Asphaltenes are defined as the fraction of crude oil soluble in aromatic solvents and insoluble in $n$-alkanes, ${ }^{1-3}$ with the molecular structure often described by a single polycyclic aromatic core with alkyl-side chains. This structure is frequently described as island-like, but for the purpose of clarity, we refer to this structure as single-core. Asphaltenes have also been found to contain multiple aromatic cores connected by covalent bonds such as aryl-aryl or alkyl-chains, which are typically referred to as aryl-linked and archipelago-like structures. ${ }^{4,5}$ This type of structure we refer to as multi-core. Asphaltenes are reported to have a hydrogen-to-carbon $(\mathrm{H} / \mathrm{C})$ ratio between $\sim 0.90$ and 1.20 and contain several heteroatoms such as sulfur, oxygen, and nitrogen (total content between $\sim 5$ and 10 wt \%) and trace amounts of metals (vanadium and nickel). ${ }^{6,7}$ While asphaltenes are frequently studied, it is increasingly clear that the behavior of asphaltenes in the bulk fluid and at interfaces (solid-liquid and liquid-liquid) is greatly influenced by the asphaltene chemistry and molecular structure.

Fractionating asphaltenes into smaller subgroups provides greater clarity on their physicochemical properties. Step-wise fractionation of asphaltenes is most commonly achieved by decreasing the solvent aromaticity to increase alkane content, preferentially precipitating progressively less soluble asphaltenes. By comparing the chemistry of precipitated and soluble asphaltenes from four different crude oils, Spiecker et al. ${ }^{8}$ found only marginal differences in composition among the four crude oils, with the precipitated fractions being slightly more aromatic with slightly higher nitrogen and oxygen contents. Changes in sulfur content between the two asphaltene fractions were found to be negligible. The most discernible changes were in the metal contents, with the precipitated fractions being more enriched in $\mathrm{Fe}, \mathrm{Ni}$, and $\mathrm{V}$.

Crude oils that exhibit severe deposition problems have been found to contain higher amounts of polarizable compounds. ${ }^{9}$ By comparing the asphaltene subfractions obtained via adsorption on $\mathrm{CaCO}_{3}$, Pinto et al. ${ }^{10}$ observed more distinct changes in the elemental composition: adsorbed asphaltenes showed higher contents of oxygen-containing compounds and decreased alkylsubstitution as compared with whole asphaltenes and weakly adsorbed fractions. Kilpatrick et al. confirmed that the asphaltene fraction stabilizing water-in-oil emulsions was more polar and acidic (higher oxygen content) and of lower double bond equivalents $(\mathrm{DBE}=$ total number of rings and double bonds involving carbon). ${ }^{11,12}$ These authors found that the stabilizing asphaltene fraction formed larger asphaltene aggregates and formed stronger bonds, ${ }^{11}$ as evidenced by neutron scattering and interfacial rheology measurements. ${ }^{8,12,13}$ Following the method of $\mathrm{Wu},{ }^{14}$ Czarnecki and co-workers ${ }^{15-17}$

Received: August 15, 2020

Revised: October 5, 2020

Published: October 21, 2020 
separated the interfacial material from crude oils and asphaltenes. Their study revealed an abundance of compounds of low aromaticity being enriched in $\mathrm{O}_{x}$ and $\mathrm{O}_{x} \mathrm{~S}_{y}$ groups ( $\mathrm{S}$ - and $\mathrm{O}$-containing functionalities) and being depleted in $\mathrm{N}$ containing functionalities. Such characteristics were also found for asphaltenes adsorbed onto calcium carbonate. ${ }^{18-20}$

Further fractionation of asphaltenes from both bitumen and heavy oil by the E-SARA method ${ }^{21-25}$ has revealed differences in the size and shape of asphaltene nanoaggregates, which correlate to the asphaltene chemistry. The emulsion stabilizing asphaltenes, referred to as interfacially active asphaltenes (IAA), account for only $\sim 2$ wt $\%$ of the whole asphaltenes (WA); the remaining, less interfacially active asphaltenes are referred to as remaining asphaltenes (RA). Chemical characterization of the IAA and RA fractions revealed differences in chemical composition, with sulfur and oxygen contents in the IAA fraction exceeding those in RA. ${ }^{24,25}$ More significantly, the abundance of different functional groups, in particular the presence of sulfoxides as a strong hydrogen-bond acceptor in IAA, contributes to the strong stabilizing (emulsion stability) potential of the IAA fraction. Recently, SANS measurements of WA extracted from bitumen and heavy oil revealed a remarkable similarity in asphaltene nanoaggregates size and shape, even though the contents of heteroatoms varied significantly. ${ }^{23}$ However, comparing IAA and RA extracted from heavy oil, similar differences in the heteroatom (sulfur and oxygen) content were observed but, crucially, the IAA fraction was enriched in sulfoxide and carbonyl groups. The higher concentration of sulfoxide groups in IAA was postulated to govern the nanoaggregate size and shape, with the IAA nanoaggregates being substantially larger and more porous than RA. ${ }^{23}$ The SANS study highlighted the possibility that the two asphaltene fractions, IAA and RA, aggregate differently, with the mechanism likely dependent on the chemistry of asphaltenes.

Although the presence of sulfoxides in crude oils has been widely debated, ${ }^{26-30}$ such uncertainty has resulted from an inability to measure sulfoxide species. Using solvent extraction, Ren et al. ${ }^{31}$ separated polar (sulfoxides) and nonpolar (sulfides and thiophenes) sulfur fractions from three different crude oils. The extracted samples were analyzed by Fourier transform-ion cyclotron resonance mass spectrometry (FT-ICR MS). The results showed a more significant abundance of the $\mathrm{O}_{1} \mathrm{~S}_{1}$ class species in the polar fraction than in the nonpolar fraction. Reduction of the polar fraction by $\mathrm{LiAlH}_{4}$ confirmed the species to be sulfoxides. The authors stated that the polar fraction of sulfur accounted for $27 \%-46 \%$ of the total sulfur, with only $0.8 \%-5.7 \%$ of the total sulfur being sulfoxides. Although the sulfoxide-to-total-sulfur content is relatively small, its presence and likely position within the asphaltene molecular structure appears to be a dominant feature governing the aggregation of asphaltene molecules.

Recent studies demonstrated that differences in aggregation tendency between structures may promote differences in ion production efficiency, which results in selective ionization of asphaltenes by atmospheric pressure photoionization (APPI). ${ }^{32}$ To fully characterize asphaltenes, an extrography fractionation method was developed, in which asphaltenes adsorbed on $\mathrm{SiO}_{2}$ were sequentially extracted using acetone, Heptol, and toluene/ tetrahydrofuran/methanol (Tol/THF/MeOH). This fractionation method extracts condensed polyaromatic structures, nonpolar alkyl-aromatic compounds, and highly polarizable species, which interact most strongly with the silanol groups of the silica gel. ${ }^{32-34}$ Characterization by APPI FT-ICR MS and gas-phase fragmentation by infrared multi-photon dissociation revealed that the fraction extracted using acetone produced $\sim 50$-fold more monomeric asphaltene ions (nonaggregated) of abundant single-core motifs. Conversely, the Tol/THF/MeOH compounds were more difficult to ionize and revealed abundant multi-core structures. These authors suggested a link between asphaltene aggregation and low ionization efficiency.

Despite extensive efforts to characterize asphaltenes, more detailed analysis of asphaltene subfractions, in particular those that strongly adsorb at interfaces, is needed to reveal determinant physicochemical properties that govern asphaltenes behaviors. High-resolution molecular characterization of asphaltenes is possible using positive-ion $(+)$ atmospheric pressure photoionization (APPI) custom-built 9.4 T FT-ICR MS and is used for the first time to study RA and IAA fractions extracted from a South American heavy crude oil. The gas-phase fragmentation via IRMPD is also performed to understand the molecular structure of the most abundant asphaltene ion generated from each fraction.

\section{MATERIALS AND METHODS}

Samples and Chemicals. Whole asphaltenes (WA) were extracted from heavy oil (Llanos region, Colombia; API $13.6^{\circ}$ ) using a standard protocol for asphaltene precipitation. ${ }^{25}$ Heavy oil $(50 \mathrm{~mL})$ was mixed with $1950 \mathrm{~mL}$ of $n$-heptane (Sigma-Aldrich) (40:1 by volume) in a $2000 \mathrm{~mL}$ beaker to precipitate asphaltenes. After $24 \mathrm{~h}$, the diluted sample was filtered using an $8 \mu \mathrm{m}$ Whatman \#2 filter paper to collect the precipitated asphaltenes, which were then washed with excess $n$ heptane until the filtrate appeared clear. Precipitated asphaltenes were then dissolved in excess toluene (HPLC-grade, Sigma-Aldrich) to remove the fine mineral solids by centrifugation at $20000 \mathrm{~g}$ for $20 \mathrm{~min}$. Asphaltenes were then dried under nitrogen, stored in airtight sealed glass vials, wrapped in aluminum foil, and refrigerated prior to their use. Prior to FT-ICR analysis, asphaltenes were further cleaned using heptane by Soxhlet extraction (Whatman, GE Healthcare, Little Chalfont, UK).

E-SARA. WA was fractionated into IAA and RA following the ESARA separation method. ${ }^{22}$ Deionized water $(10 \mathrm{~mL})$ was added to $100 \mathrm{~mL}$ of WA-in-toluene $(10 \mathrm{~g} / \mathrm{L})$; the mixture was homogenized (T18 Ultra-Turrax, IKA) at $13000 \mathrm{rpm}$ for $15 \mathrm{~min}$. The emulsion was gently agitated for $24 \mathrm{~h}$ and subsequently centrifuged at $11000 \mathrm{rpm}$ (Heraeus Megafuge 16 Centrifuge, ThermoFisher Scientific) to separate the asphaltene-stabilized water droplets from the continuous oil phase. The supernatant was then removed without disturbing the "emulsion cake", and an equal volume of fresh toluene was added and shaken to remove any loosely bound asphaltenes at the oil-water interface. Following six consecutive toluene washes, the emulsion droplets were transferred to a preweighed glass beaker and left for $12 \mathrm{~h}$ in a vacuum oven at $60^{\circ} \mathrm{C}$. This process is aimed to evaporate deionized water and the remaining solvent. Using this procedure, $1 \mathrm{~g}$ of WA heavy oil (asphaltene content of the heavy oil was $\sim 25 \mathrm{wt} \%$ ) yielded $\sim 15 \mathrm{mg}$ of IAA, confirming that the fraction of asphaltenes partitioned at the oil-water interface was $\sim 1.5$ wt $\%$ of WA.

Extrography Fractionation. The RA and IAA fractions were further separated using the extrography method, ${ }^{32}$ which assisted the selective separation of asphaltenes with a high production efficiency of nonaggregated ions in APPI (high monomer ion yield, MIY) to facilitate mass spectral characterization of difficult-to-ionize species. Asphaltenes were dissolved in dichloromethane (DCM, J.T. Baker) at $0.2 \mathrm{~g} / \mathrm{L}$ and mixed for $1 \mathrm{~h}$ with chromatographic-grade silica gel (Fluka Analytical, 70-230 mesh, pore size $60 \AA$ ) at $5 \mathrm{mg}$ of asphaltenes per $1 \mathrm{~g}$ of $\mathrm{SiO}_{2}$ mass ratio. The mixture was completely dried under nitrogen $(8$ h) and then Soxhlet extracted with acetone for $24 \mathrm{~h}$ (HPLC-grade, J.T. Baker), separating species of high monomer ion yield, which typically bias mass spectrometry analyses of asphaltenes due to ion suppression. The mixture of the remnant asphaltenes + silica gel was again dried and 

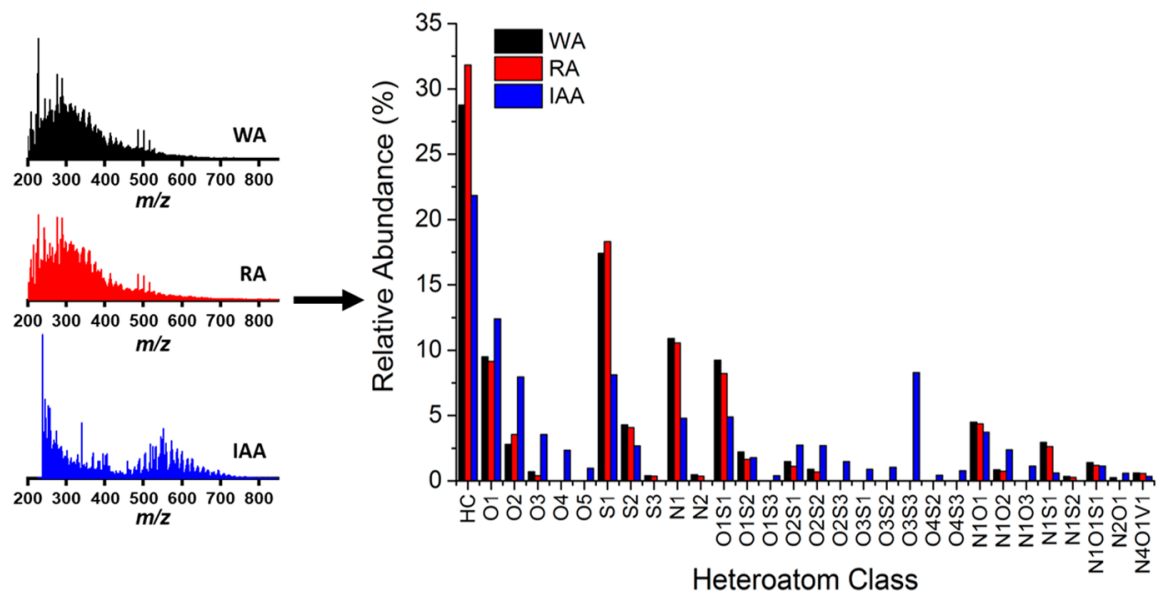

Figure 1. APPI(+) FT-ICR MS of WA, RA, and IAA extracted from a South American heavy oil (left), with the relative abundance (\%) of assigned heteroatom classes for each of the three asphaltene samples (right).

Soxhlet extracted with Heptol (1:1 v/v $n$-heptane and toluene) to separate nonpolar alkyl-aromatic compounds. ${ }^{35-37} \mathrm{~A}$ third extraction was conducted using a mixture of toluene, tetrahydrofuran (THF), and methanol $(\mathrm{MeOH})$ in a volume ratio of 2:2:1 to extract the polarizable asphaltenes that interact with the silica gel via hydrogen bonding. ${ }^{38-40}$ Yields for each extraction and monomer ion yields (MIY) are provided in Figure 6.

Positive-Ion Atmospheric Pressure Photoionization 9.4 T Fourier Transform lon Cyclotron Resonance Mass Spectroscopy ((+)APPI 9.4 T FT-ICR MS) and Infrared multi-photon Dissociation (IRMPD). All asphaltene samples were diluted to 100 $\mu \mathrm{g} / \mathrm{mL}$ in toluene (HPLC-grade, Sigma-Aldrich) and directly infused into a Thermo-Fisher Ion Max APPI source (Thermo-Fisher Scientific, Inc. $)^{41}$ at a vaporizer temperature of $350^{\circ} \mathrm{C}$ and a sample flow rate of 50 $\mu \mathrm{L} / \mathrm{min}$. Sample nebulization was assisted by $\mathrm{N}_{2}$ gas. Gas-phase neutrals were photoionized by $10.2 \mathrm{eV}$ photons from a Krypton lamp. A custom-built 9.4-T Fourier transform ion cyclotron resonance mass spectrometer was used to characterize the ionized asphaltene species, with 100 scans averaged per sample. ${ }^{42}$ The RA and WA samples were characterized over an $m / z$ range of 200-1000, and the IAA sample was measured over an $m / z$ range of $240-1000$. For IAA, the removal of low $\mathrm{m} / \mathrm{z}$ data was performed using a quadrupole mass filter to improve the signal of higher molecular weight compounds in the IAA sample, as a high abundance of low $\mathrm{m} / z$ ions derived from toluene clusters was detected. Due to the high abundance of low $m / z$ ions derived from toluene clusters, the removal of low $m / z$ data was performed using a quadrupole mass filter to improve the signal of higher molecular weight compounds in the IAA sample. Data collection was performed using a modular ICR data acquisition system (PREDATOR). ${ }^{42-44}$ Positive ions were accumulated externally for $100-10000 \mathrm{~ms}$ in a radio frequency (rf)-only octopole and collisionally cooled with helium prior to transfer to a custom dynamically harmonized FT-ICR cell. One hundred time-domain acquisitions were coadded, Hanning-apodized, zero-filled, and Fourier transformed. Analysis software assisted frequency transformation to $m / z$ by the quadrupolar electric trapping potential approximation, peak detection $(>6 \sigma$ baseline root-meansquare (RMS) noise), and internal calibration by extended homologous alkylation series. Molecular formula assignment and data visualization by iso-abundance-contoured plots of double bond equivalents (DBE) vs carbon number were obtained using the PetroOrg N-18.3 software. ${ }^{45}$ Unless otherwise stated, the data is the combination of radical and protonated ions, with mass errors less than $0.2 \mathrm{ppm}$ and root-meansquare errors below $0.14 \mathrm{ppm}$. The data was also processed to only contain ions following a homologous series of at least five and peaks identified to contain up to six sulfur, oxygen, and/or nitrogen atoms. The molecular formula assignment was performed using an iterative process, in which low-heteroatom content ions are assigned first to decrease the possibility of misassignments. The DBE is equal to the number of carbon involving rings and double bonds in a given structure and is calculated from the assigned molecular formulas using the equation $\mathrm{DBE}=\mathrm{C}-\frac{H}{2}+\frac{N}{2}+1$, where $C$ is the number of carbon atoms, $H$ is the number of hydrogens, and $N$ is the number of nitrogen atoms in each assigned molecular formulas.

Detected ions not observed as a homologous series (compounds with the same DBE but varying carbon number) of at least five members and classes with a relative abundance (ra \%) below $0.15 \%$ were excluded from the analysis. More than 20000 molecular formulas for WA, RA, and IAA were assigned. The assigned formulas were compared in terms of their relative abundance, which is expressed as a percentage of the identified heteroatom classes.

Isolation of precursor ions within a $4 \mathrm{Da}$ range at $\mathrm{m} / z 450$ and 650 were selected for fragmentation via a mass-filter quadrupole, followed by multi-notch stored waveform inverse Fourier transform (SWIFT) using a method previously described. ${ }^{5,32-34}$ The multi-notch SWIFT process helps to isolate ions with a mass defect $<0.25000 \mathrm{Da}$ (DBE $>$ 15-18), which remain in the ICR for subsequent fragmentation at a remarkably low pressure $\left(<1 \times 10^{-10}\right.$ Torr $)$. The precursor ions were subjected to infrared radiation $(\lambda=10.6 \mu \mathrm{m}, 40 \mathrm{~W}$ irradiation, Synrad $\mathrm{CO}_{2}$ laser, Mukilteo, WA) for 50-2000 ms. The produced fragments were analyzed by conventional FT-ICR MS. The boundary between single-core and multi-core structures was determined as the difference between the abundance-weighted (AW) average DBE value of the precursor ions and their AW standard deviation. The boundary is identified by the red dashed lines in the IRMPD data.

\section{RESULTS AND DISCUSSION}

WA, RA, and IAA Fractions. Compositional similarity between WA and RA fractions was observed. This is not surprising since WA is composed of $\sim 98.5$ wt \% of RA. Therefore, detailed comparisons of WA to RA and IAA are not presented; however, for reference, the WA data are provided in the Supporting Information (iso-abundance-contoured plots of DBE vs carbon number for different heteroatom classes, Figures S1-S7).

Figure 1 compares the relative abundance (\%) of different heteroatom classes (elemental composition assigned from mass spectral peaks) in RA and IAA. WA is also included to highlight the similarity between WA and RA fractions. Heteroatom classes were assigned on the basis of the elemental compositions, for example, species containing one nitrogen atom were assigned to the $\mathrm{N}_{1}$ class and species containing no heteroatoms were assigned to the $\mathrm{HC}$ class. It is clear that the heteroatom content differentiates the E-SARA fractions, with $\sim 53 \%$ of IAA containing two or more heteroatoms, compared with just 

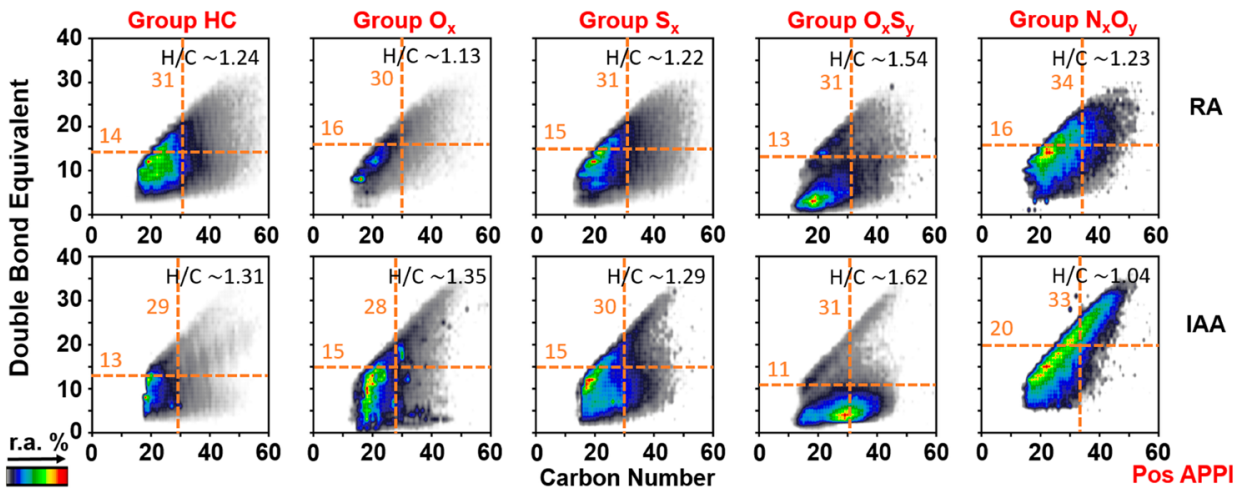

Figure 2. Iso-abundance-contoured plots of DBE vs carbon number for different heteroatom groups of RA (top row) and IAA (bottom row). The relative abundance (\%) is shown by the color key with gray and red representing low and high relative abundances, respectively. Dashed lines represent the AW average DBE and carbon number. The AW average $\mathrm{H} / \mathrm{C}$ mass ratio is shown as the inset.

$\sim 30 \%$ for RA (Figure 1). The molecular weight distributions for all three samples are shown for the $m / z$ range $200-850$.

The WA and RA samples revealed similar molecular weight distributions, which spanned from 200 to $\sim 650 \mathrm{Da}$, and a peak intensity in the region of $300 \mathrm{Da}$ (average molecular weights of 440 and $441 \mathrm{Da}$, respectively). For IAA, a clear bimodal distribution was observed, with the two peak intensities measured in the region of 250 and $550 \mathrm{Da}$ and the molecular weight distribution measured up to $\sim 750 \mathrm{Da}$ (average molecular weight of $447 \mathrm{Da}$ ). Similar molecular weight distributions for RA and IAA were previously found when measured using nanostructure-assisted laser desorption mass spectroscopy (NALDI-MS), ${ }^{23}$ although the values reported by NALDI-MS were slightly higher. The smaller molecular weight values reported by APPI FT-ICR MS results from using APPI, which produces ions with a higher internal energy, reducing the possibility of detecting low-order aggregates (dimers, trimers, etc.). Larger aggregates are not observed in the mass spectra because the ICR cell is tuned for detecting compounds with $\mathrm{m} / z$ between 100 and 1500. The bimodal molecular weight distribution of IAA may hint at the mechanism for interfacial film formation and growth, with a bimodal distribution possibly supporting a two-step adsorption process. While this is currently speculative, it suggests that more characterization of the smaller and larger IAA fractions could provide further insights, although this is currently beyond the scope of the current study.

Compound classes can be sorted into heteroatom groups. For instance, the classes S1, S2, S3, etc., belong to the group $S_{x}$. Comparison of the relative abundance of each heteroatom group for IAA and RA (Figure S8) indicated that the $\mathrm{HC}, \mathrm{N}_{x}, \mathrm{~S}_{x}$, and $\mathrm{N}_{x} \mathrm{~S}_{y}$ groups were depleted in the IAA fraction. Moreover, IAA was found to be enriched in $\mathrm{O}_{x}$ groups, particularly, compounds with a high content of oxygen atoms, such as $\mathrm{O}_{3-5}, \mathrm{~N}_{1} \mathrm{O}_{2-3}$ $\left(\mathrm{N}_{x} \mathrm{O}_{y}\right)$, and $\mathrm{O}_{2-4} \mathrm{~S}_{1-3}\left(\mathrm{O}_{x} \mathrm{~S}_{y}\right)$ heteroatom classes. These findings agree with those of the previous studies of Czarnecki and co-workers who confirmed enrichment of $\mathrm{O}_{x}$ and $\mathrm{O}_{x} \mathrm{~S}_{y}$ groups in the interfacial material that stabilizes water-in-crude oil emulsions. ${ }^{15-17}$ The enrichment of $\mathrm{O}_{x}$ and $\mathrm{O}_{x} \mathrm{~S}_{y}$ groups is also consistent with the results of bulk elemental composition analysis of IAA and RA fractions: $\mathrm{S}$ and $\mathrm{O}$ contents of IAA (heavy crude oil) were found to be $4.43 \mathrm{wt} \%(\mathrm{~S})$ and $4.57 \mathrm{wt} \%$ (O), compared with 3.45 wt \% (S) and 2.41 wt \% (O) for RA. ${ }^{23}$

The relative abundance of vanadyl porphyrins $\left(\mathrm{N}_{4} \mathrm{O}_{1} \mathrm{~V}_{1}\right)$ in both RA and IAA fractions was found to be small, with less $\mathrm{N}_{4} \mathrm{O}_{1} \mathrm{~V}_{1}$ being measured in the IAA fraction. While recent studies have highlighted the importance of porphyrins in stabilizing emulsions, ${ }^{46}$ it is worth noting that the lack of detection of vanadyl porphyrins in MS may be due to the strong aggregation tendency of the IAA fraction, ${ }^{47,48}$ which decreases its monomer ion yield in APPI and precludes detection of certain compound classes. Vanadyl porphyrins that were detected in all samples revealed similar ranges of carbon number ( 26-36) and DBE ( 18-22). The molecular composition (DBE vs carbon number plots) is shown in the Supporting Information, Figure S7.

Heteroatom groups can be further analyzed by the isoabundance-contoured plots of DBE vs carbon number. Figure 2 presents the DBE vs carbon number plots of $\mathrm{HC}, \mathrm{O}_{x}, \mathrm{~S}_{x}, \mathrm{O}_{x} \mathrm{~S}_{y}$, and $\mathrm{N}_{x} \mathrm{O}_{y}$ groups for RA and IAA.

The iso-abundance-contoured plots (Figure 2) confirmed different molecular compositions of RA and IAA. Except for the $\mathrm{N}_{x} \mathrm{O}_{y}$ group, which accounts for $\sim 5 \%$ of the heteroatomcontaining compounds in RA and IAA, all other heteroatom groups exhibited a higher $\mathrm{H} / \mathrm{C}$ mass ratio in IAA than in RA. The AW average $\mathrm{H} / \mathrm{C}$ mass ratios for the RA and IAA fractions were 1.25 and 1.39. Using the same extraction procedure and the same batch of heavy crude oil, slightly lower $\mathrm{H} / \mathrm{C}$ mass ratios were measured by elemental analysis $(\mathrm{RA}=1.04$ and IAA $=$ 1.21). ${ }^{23}$ Although other studies have found APPI to underestimate asphaltene $\mathrm{H} / \mathrm{C}$ ratios compared to elemental analysis, ${ }^{49}$ it is likely that the discrepancy in the current study results from the sample consisting of lower DBE, oxygen-rich ions, which are more prone to gas phase/proton transfer reactions during dopant-assisted APPI, resulting in efficient ionization of compounds with high proton affinities. ${ }^{34,50-52}$ Conversely, highly aromatic and oxygen deficient samples are more prone to ionize as radical cations, which would result in lower MS-derived $\mathrm{H} / \mathrm{C}$ ratios. In our case, there were higher MS-derived $\mathrm{H} / \mathrm{C}$ ratios than bulk elemental analysis. Regardless, the trend observed by MS was consistent with bulk elemental analysis; the IAA fraction is less aromatic than RA. Apart from the $\mathrm{N}_{x} \mathrm{O}_{y}$ group, the average carbon number and AW average $\mathrm{DBE}$ of the heteroatom groups in IAA were either equivalent or lower than in RA.

The AW average DBE values for $\mathrm{N}_{x} \mathrm{O}_{y}$ in RA and IAA were 16 and 20 , with less than $1 \%$ of the detected ions having a DBE $>30$ (highly aromatic species) for RA and 3.6\% for IAA. A Pearson correlation analysis between $\mathrm{DBE}$ and carbon number for the $\mathrm{N}_{x} \mathrm{O}_{y}$ group revealed correlation coefficients of 0.52 and 0.72 for $\mathrm{RA}$ and IAA, respectively. A narrower distribution (DBE vs 
carbon number) and high DBE values for IAA suggests that the $\mathrm{N}$-containing species are most likely associated with large polyaromatic cores.

Heteroatomic functionalities of the $\mathrm{O}_{x} \mathrm{~S}_{y}$ group in IAA are less likely to be contained within aromatic cores, especially in species containing multiple oxygen atoms such as $\mathrm{O}_{3} \mathrm{~S}_{3}$ and $\mathrm{O}_{4} \mathrm{~S}_{3}$, which feature remarkably low DBE values (Figure S5). For RA, only heteroatom classes up to $\mathrm{O}_{2} \mathrm{~S}_{2}$ (Figure S2) were detected at levels above the measurement threshold (abundance $>6 \sigma$ ), whereas for IAA, the $\mathrm{O}_{x} \mathrm{~S}_{y}$ group included species up to $\mathrm{O}_{4} \mathrm{~S}_{3}$ (Figure S5). Further analysis of the $\mathrm{O}_{x}$ and $\mathrm{O}_{x} \mathrm{~S}_{y}$ groups showed a decrease in the AW average DBE with increasing $\mathrm{O}$ content. For example, for the $\mathrm{O}_{x}$ group, the AW average DBE for RA and IAA decreased from $16\left(\mathrm{O}_{1}\right)$ to $12\left(\mathrm{O}_{3}\right)$ and $15\left(\mathrm{O}_{1}\right)$ to $9\left(\mathrm{O}_{5}\right)$, respectively. Similar changes in the AW average $\mathrm{DBE}$ of $\mathrm{O}_{x} \mathrm{~S}_{y}$ group for RA and IAA were also observed (Figures S2, S3 and S5, Supporting Information). A reduction in the AW average DBE for higher-order oxygen containing groups is consistent with the results from previous studies where higher-order oxygen containing groups were associated with lower aromaticity asphaltene structures. ${ }^{34,53-55}$

The APPI ion source produces protonated molecules $([\mathrm{M}+$ $\left.\mathrm{H}]^{+}\right)$and radical cations $\left(\mathrm{M}^{+\bullet}\right),{ }^{50}$ with separate iso-abundancecontoured plots of DBE vs carbon number revealing differences in their compositional range. In Figure 2, it was shown that the $\mathrm{O}_{x} \mathrm{~S}_{y}$ group of IAA had a high abundance of species below the AW average DBE of 11 . Comparing the protonated compounds $\left([\mathrm{M}+\mathrm{H}]^{+}\right)$and radical cations $\left(\mathrm{M}^{+} \bullet\right)$ for the heteroatom class $\mathrm{O}_{1} \mathrm{~S}_{2}$ (chosen due to its high relative abundance), Figure 3

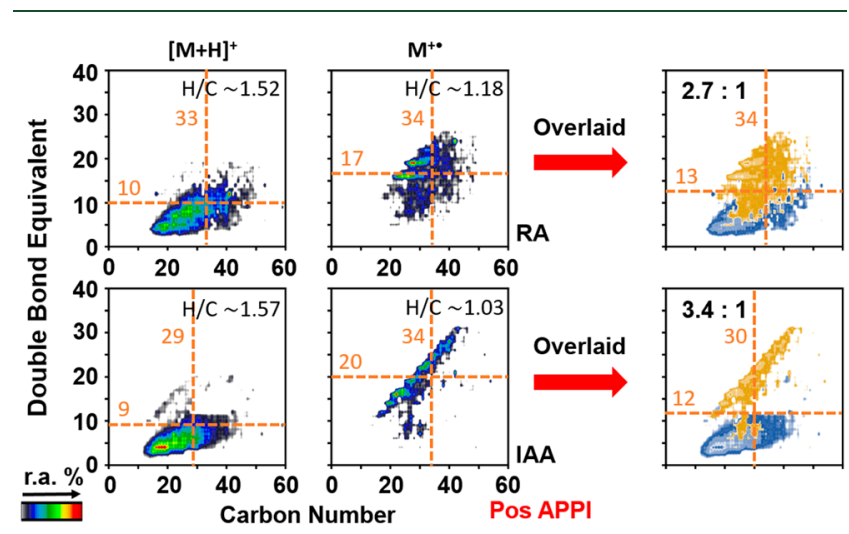

Figure 3. Iso-abundance-contoured plots of $\mathrm{DBE}$ vs carbon number of $\mathrm{O}_{1} \mathrm{~S}_{2}$ protonated $\left([\mathrm{M}+\mathrm{H}]^{+}\right)$and radical $\left(\mathrm{M}^{+\bullet}\right)$ cations for RA (top row) and IAA (bottom row). Dashed lines represent AW average DBE and carbon number. The $\mathrm{AW}$ average $\mathrm{H} / \mathrm{C}$ mass ratio is shown inset. Overlaid iso-abundance-contoured plots with protonated and radical species being highlighted by the blue-shaded and yellow-shaded regions, respectively. The protonated-to-radical ratio is shown in each corresponding plot.

shows that the protonated species $\left(\mathrm{O}_{1} \mathrm{~S}_{2}[\mathrm{M}+\mathrm{H}]^{+}\right)$have an AW average $\mathrm{DBE} \leq 10$ and feature a compositional range at lower DBE than the radical cations. A better separation of protonated and radical cation species was seen for the IAA fraction, as is clearly shown by the overlaid iso-abundance-contoured plots in Figure 3, as well as being confirmed by the greater difference in AW average DBE values: $\mathrm{LAA}=11$ and $\mathrm{RA}=7$. Moreover, the abundance ratio of protonated-to-radical cations $\left([\mathrm{M}+\mathrm{H}]^{+} /\right.$ $\mathrm{M}^{+} \bullet$ ) was greater in IAA than in RA (IAA = 3.4 and RA = 2.7), demonstrating that IAA compounds preferentially ionize via protonation, which correlates with a higher tendency to hydrogen-bond in solution. Furthermore, the protonated species of both $\mathrm{RA}$ and IAA reveal a higher $\mathrm{H} / \mathrm{C}$ mass ratio $(\mathrm{RA} \sim 1.52$; $\mathrm{IAA} \sim 1.57)$ than the radical cations $(\mathrm{RA} \sim 1.18$; IAA 1.03). In summary, the IAA fraction is enriched in low aromatic molecular structures, which have a tendency to form hydrogen bonds.

The relative abundance of the $\mathrm{O}_{x} \mathrm{~S}_{y}$ group in RA and IAA is $11.7 \%$ and $25.4 \%$, representing a significant difference between the two asphaltene fractions (the relative abundances of $\mathrm{HC}, \mathrm{O}_{x}$, $\mathrm{S}_{x}, \mathrm{~N}_{x}, \mathrm{O}_{x} \mathrm{~S}_{y}, \mathrm{~N}_{x} \mathrm{O}_{y}, \mathrm{~N}_{x} \mathrm{O}_{y} \mathrm{~S}_{z}$, and $\mathrm{N}_{x} \mathrm{O}_{y} \mathrm{~V}_{z}$ groups are shown in Figure S8). Given this difference and the fact that IAA contains higher amounts of $S$ and $O$, with a likely significant enrichment in the $\mathrm{S}=\mathrm{O}$ functional group, ${ }^{21-25}$ it can be postulated that the water-in-oil stabilizing asphaltenes are predominantly composed of low aromaticity molecular structures containing $\mathrm{O}_{x} \mathrm{~S}_{y}$ groups that readily ionize as protonated molecules. These "atypical" structures are classified as asphaltenes as a result of their functionality and polarity rather than high aromaticity.

Fragmentation of IAA and RA. In an attempt to determine the predominant structure (single-core or multi-core) of IAA and RA, precursor ions of relatively high molecular weight $(\mathrm{m} / \mathrm{z}$ 650 ), where multi-core structures are likely to be observed (if they are present), ${ }^{56-58}$ were isolated via mass-resolving quadrupole and SWIFT multi-notch and fragmented by IRMPD (see Figure 4 for the fragmentation spectra). Fragmentation data of lower molecular weight $(\mathrm{m} / z$ 450) ions are shown in the Supporting Information to highlight the sensitivity of the single-core/multi-core predominance on molecular weight. IRMPD yields the most stable species by removing alkyl-chains and cleaving the alkyl bridges between aromatic cores, leaving stable aromatic cores unfragmented. ${ }^{32,59}$ Greater fragmentation of IAA was verified by considering the abundance of species below and above a threshold of $m / z 400$. This threshold was chosen as alkyl-substituted low ring number polycyclic aromatic hydrocarbons (PAHs), such as naphthalene, phenanthrene, pyrene, and dibenzothiophene, have masses below $\mathrm{m} / z 400$. The abundance ratio of species below $\mathrm{m} / z 400$ was 1.45 and 2.13 for RA and IAA, confirming greater fragmentation of IAA. While this supports the view that IAA contains smaller cores (lower $M_{\mathrm{w}}$ ), which are aryl-linked or linked by alkyl-chains, the increased fragmentation of IAA may also result from the higher oxygen content, with carboxylic acid, ketone, and sulfoxide functional groups known to absorb infrared light more efficiently. ${ }^{34}$

In fragmentation, single-core structures follow a dealkylation pattern, whereby only the carbon number decreases, whereas multi-core structures lose both the carbon number and DBE. The fragmentation patterns for both RA and IAA show a reduction in DBE and carbon number, signifying the presence of multi-core structures in each fraction. Dealkylation is more evident for RA at DBE 25 and 30, suggesting a greater fraction of single-core motifs. Fragmentation of IAA led to abundant low DBE (10) and low carbon number (16) fragment ions, whereas RA revealed fragments with intermediate DBE (30) and carbon numbers (44). These intermediates likely result from dealkylation of large aromatic structures (single-core) and fragmentation of multi-core structures. To reflect the abundance of single-core and multi-core motifs in IAA and RA, we define a boundary between these two structures (identified by the red line in Figure 5). The boundary is determined by subtracting the AW standard deviation from the AW average DBE value for the assigned precursor ions, ${ }^{32}$ with the boundary at DBE of 20 and 18 for RA and IAA. The fragment ions with DBE values below 

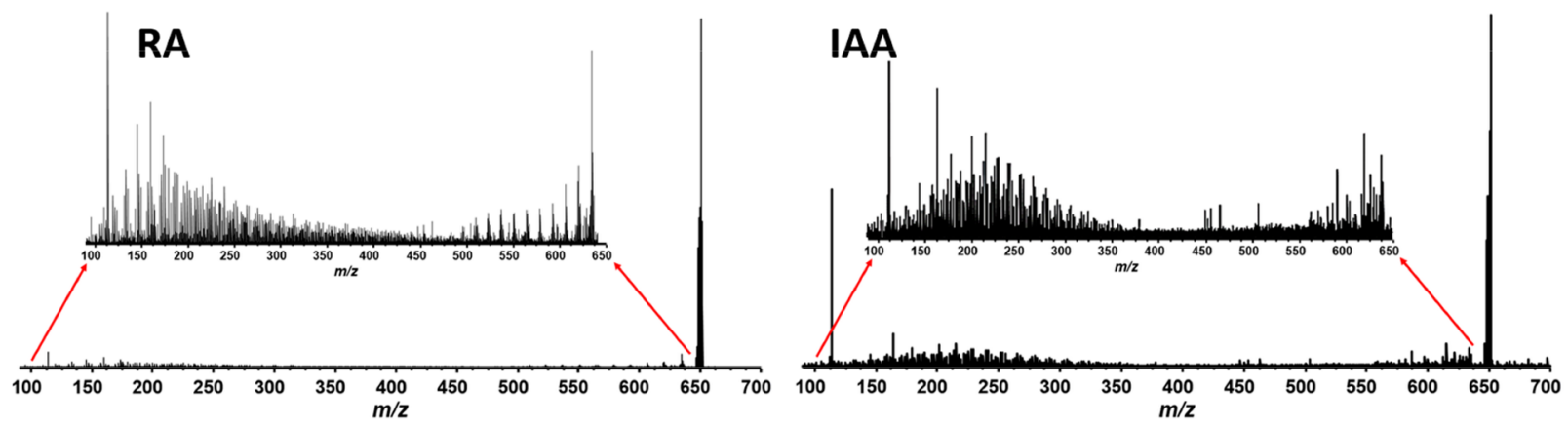

Figure 4. Mass spectra of the isolated precursor ions $(\mathrm{m} / z$ 650) (below spectra) and fragment ions detected following irradiation of the precursor ions (above spectra) by IRMPD for RA (left) and IAA (right).

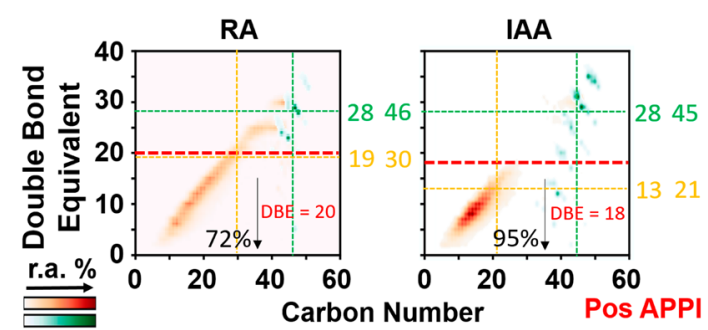

Figure 5. Combined iso-abundance-contoured plots of DBE vs carbon number of the precursor and fragment ions from RA (left) and IAA (right). The precursor and fragmented ions are identified by the green and orange color maps, respectively. Green and orange lines represent the AW average DBE (first number) and carbon number (second number) for the precursor and fragment ions. The red line represents the boundary to identify between single-core (above) and multi-core (below) structures, with the percentage value (inset) confirming the abundance of fragment ions representing multi-core motifs.

the boundary are likely produced from fragmenting multi-core structures, while those fragment ions with DBE values above the boundary are likely produced from fragmenting single-core motifs. The abundance of fragment ions below the boundary was $72 \%$ for RA and $95 \%$ for IAA, confirming that the high DBE precursor ions of IAA with $\mathrm{m} / z 650$ are almost entirely multicore structures, whereas the RA fraction is a mixture of singlecore and multi-core motifs. A greater abundance of multi-core motifs in IAA likely supports our recent SANS data, which showed that the IAA fraction form larger and more porous nanoaggregates compared to the RA fraction. ${ }^{23}$ It was speculated that the larger nanoaggregates were formed due to the presence of more functional groups (carbonyl and sulfoxide), which can now be further understood by the differences in the molecular structure of the two asphaltene subfractions. These findings agree with a molecular mechanics study, ${ }^{60}$ which showed multi-core asphaltenes form more complex (bridged and tangled) aggregates.

It is possible to discern "typical" structures of RA and IAA fractions from the changes in the AW average DBE values and carbon numbers. For RA and IAA, the AW average DBE values decreased from 46 to 30 (RA) and 45 to 21 (IAA) and the carbon numbers decreased from 28 to 19 (RA) and 28 to 13 (IAA). Such fragmentation represents a reduction in the molecular weight by $\sim 34 \%$ and $\sim 53 \%$ for RA and IAA. For complex mixtures, the reduction in molecular weight can occur through a number of different fragmentation pathways: (i) dealkylation; (ii) splitting of two equally sized aromatic cores; (iii) splitting of two aromatic cores of different size; and (iv) splitting of multiple aromatic cores. On the basis of the changes in molecular weight and the abundance of fragment ions, we postulate that RA is composed of a mixture of single-core and multi-core motifs, while IAA is predominantly multi-core structures. Figures S13 and S14 show the mass spectra and iso-abundance-contoured plots of DBE vs carbon number for the fragmented ions of RA and IAA from precursor ions of $\mathrm{m} / \mathrm{z}$ 450. The fragmentation patterns were found to be similar with most of the fragmented ions generated from single-core motifs $(\mathrm{RA}=62 \%, \mathrm{IAA}=73 \%)$. A greater abundance of single-core structures for the lower molecular weight species supports the theory that a carbon limit exists for asphaltenes, where the further addition of carbon leads to the formation of multi-core structures, as peri-condensation becomes less favored/likely. ${ }^{61}$

With a higher abundance of $\mathrm{O}_{x} \mathrm{~S}_{y}$ in IAA than RA, fragmentation of the $\mathrm{O}_{x} \mathrm{~S}_{y}$ precursor ions $(\mathrm{m} / z$ 650) was compared (see Figure S15). The RA fraction showed significant dealkylation, with only $39 \%$ of the fragment ions determined to be multi-core motifs. Fragmentation of the IAA precursor ion led to a greater reduction in the AW average DBE and carbon number, with $62 \%$ of the fragment ions resulting from multi-core structures.

To estimate the likely location of heteroatoms in RA and IAA, $\mathrm{N} / \mathrm{C}, \mathrm{O} / \mathrm{C}$, and $\mathrm{S} / \mathrm{C}$ ratios before and after IRMPD fragmentation were compared and summarized in Table 1 . We

Table 1. Comparison of Heteroatom (N, O, and S) to Carbon Ratio of $m / z 650$ Ions before and after Fragmentation by IRMPD

\begin{tabular}{llll}
\multicolumn{1}{c}{ sample } & $\mathrm{N} / \mathrm{C}$ & $\mathrm{O} / \mathrm{C}$ & $\mathrm{S} / \mathrm{C}$ \\
precursor RA & 0.0126 & 0.0177 & 0.0166 \\
fragment RA & 0.0163 & 0.0141 & 0.0189 \\
\% change & 29.7 & -20.2 & 14.2 \\
precursor IAA & 0.0152 & 0.0367 & 0.0253 \\
fragment IAA & 0.0131 & 0.0156 & 0.0207 \\
\% change & -13.9 & -57.5 & -17.9 \\
\hline
\end{tabular}

assume that strongly negative values are associated with a reduction in heteroatom content as a result of the heteroatoms being located in aliphatic side chains and thus not detected following IRMPD, because the charge most likely remains in the aromatic cores. Strongly positive values are associated with heteroatoms more likely to be found in aromatic cores, as carbon is lost in the aliphatic side chains but the heteroatom content is retained. The data shows that in both RA and IAA, $\mathrm{N}$ is most likely found within aromatic cores and $\mathrm{O}$ is most likely located in aliphatic chains. This supports our earlier finding that $\mathrm{N}$ - 


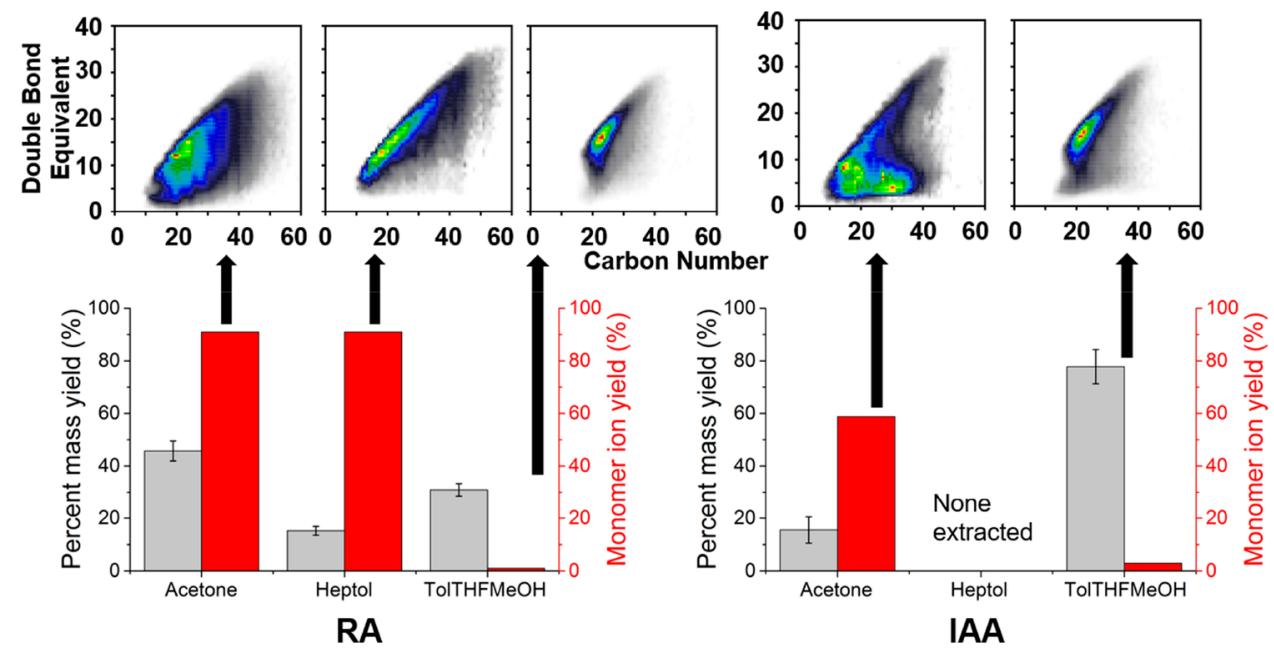

Figure 6. Mass yield (\%; gray bar) and monomer ion yield (\%; red bar) for RA (left) and IAA (right) fractions separated by the extrography method. The iso-abundance-contoured plots of DBE vs carbon number for each sample are provided above.

Table 2. Summary of Parameters Used for Model Structure Determination of RA and IAA ${ }^{a}$

\begin{tabular}{|c|c|c|c|c|c|}
\hline & \multicolumn{5}{|c|}{ RA } \\
\hline & $\mathrm{HC}$ & $\mathrm{N}_{x}$ & $\mathrm{O}_{x}$ & $\mathrm{O}_{x} \mathrm{~S}_{y}$ & $\mathrm{~S}_{x}$ \\
\hline abundance in fraction & $32 \%$ & $11 \%$ & $13 \%$ & $12 \%$ & $23 \%$ \\
\hline $\mathrm{C} \#$ and $\mathrm{DBE}$ & 24 and 10 & 23 and 12 & 21 and 12 & 19 and 3 & 22 and 12 \\
\hline reduction in C\# and DBE by IRMPD & $75 \%$ and $82 \%$ & $\mathrm{n} / \mathrm{a}$ & $8 \%$ and $12 \%$ & $9 \%$ and $14 \%$ & $69 \%$ and $64 \%$ \\
\hline \multirow[t]{5}{*}{ functional groups } & none & pyridine $^{64}$ & carbonyl $^{18}$ & carbonyl $^{18}$ & thiophene $e^{26,27,66}$ \\
\hline & & & carboxylic acid ${ }^{65}$ & carboxylic acid ${ }^{65}$ & sulfide $^{26,27,66}$ \\
\hline & & & & thiophene $e^{26,27,66}$ & sulfone $\mathrm{e}^{26,27,66}$ \\
\hline & & & & sulfoxide ${ }^{26,27,66}$ & \\
\hline & & & & sulfone $e^{26,27,66}$ & \\
\hline \multirow[t]{4}{*}{ heteroatom content } & \multirow{2}{*}{\multicolumn{5}{|c|}{$\begin{array}{l}\text { - } \sim 30 \% \text { of fraction contains no heteroatoms } \\
\text { - }>35 \% \text { of fraction contains only } 1 \text { heteroatom (S1, N1, or O1) }\end{array}$}} \\
\hline & & & & & \\
\hline & \multicolumn{5}{|c|}{ IAA } \\
\hline & $\mathrm{HC}$ & $\mathrm{N}_{x} \mathrm{O}_{y}$ & $\mathrm{O}_{x}$ & $\mathrm{O}_{x} \mathrm{~S}_{y}$ & $\mathrm{~S}_{x}$ \\
\hline abundance in fraction & $22 \%$ & $8 \%$ & $27 \%$ & $25 \%$ & $11 \%$ \\
\hline C\# and DBE & 19 and 8 & 26 and 13 & 18 and 12 & 29 and 4 & 20 and 12 \\
\hline reduction in C\# and DBE by IRMPD & $73 \%$ and $79 \%$ & $10 \%$ and $14 \%$ & $74 \%$ and $71 \%$ & $64 \%$ and $40 \%$ & $71 \%$ and $71 \%$ \\
\hline \multirow[t]{5}{*}{ functional groups } & none & pyridine $^{64}$ & carbonyl $^{18}$ & carbonyl $^{18}$ & thiophene $e^{26,27,66}$ \\
\hline & & & carboxylic acid ${ }^{65}$ & carboxylic acid ${ }^{65}$ & sulfide $26,27,66$ \\
\hline & & & & thiophene $e^{26,27,66}$ & sulfone $\mathrm{e}^{26,27,66}$ \\
\hline & & & & sulfoxide ${ }^{26,27,66}$ & \\
\hline & & & & sulfone $\mathrm{e}^{26,27,66}$ & \\
\hline
\end{tabular}

heteroatom content $\quad \bullet \sim 50 \%$ of fraction contains polyheteroatomic species

- oxygen-containing species noticeably abundant

${ }^{a_{T}}$ The reduction in carbon number $(\mathrm{C \#})$ and DBE was taken as the difference between modal values for the precursor and fragmented ions, with $\mathrm{n} /$ a reported for heteroatom groups where precursor ions were not detected.

containing species have a high $\mathrm{DBE}$ and therefore are highly aromatic (see Figures S1, S6, and S7).

Fractionation by Extrography. Further fractionation of RA and IAA was made using the extrography method to yield asphaltene fractions enriched in single-core and multi-core structures. By using acetone, Heptol 1:1, and Tol/THF/MeOH as extracting solvents, it is possible to selectively extract highly aromatic compounds, nonpolar species, and polar molecules interacting via hydrogen bonding with the silica gel. ${ }^{5,32}$ Figure 6 shows the mass yield (\%) and the monomer ion yield (\%; MIY) for both RA and IAA eluted using the three solvents, with the mass yield reflecting the solubility and the MIY the ionization efficiency of each fraction.
Through comparing the mass yields of the extrography fractions, the data supports our earlier finding and confirms that $\mathrm{RA}$ is a mixture of abundant single-core and multi-core structures, while IAA is enriched in multi-core motifs. Acetone elution led to mass yields of $45.7 \pm 3.8 \%$ and $15.5 \pm 5.0 \%$ for RA and IAA, respectively. The higher monomer ion yield of RA (acetone eluted) compared to IAA is in good agreement with the ion production efficiency of single-core and multi-core structures, $^{5,32-34,56}$ with the low monomer ion yield of IAA resulting from a predominance of highly aggregated multi-core species during APPI ionization. While extrography has previously been used to separate highly aromatic compounds and polar molecules, the prior fractionation of WA by the E- 


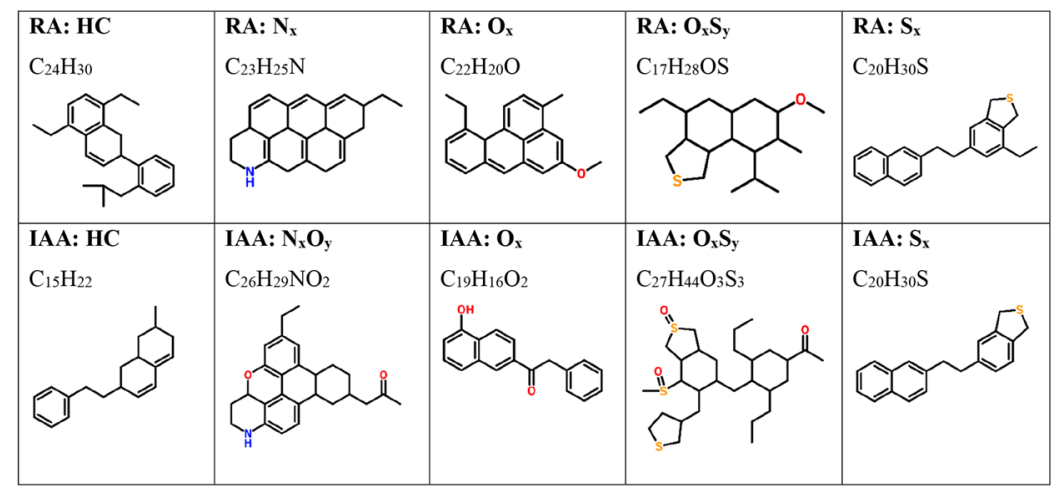

Figure 7. Proposed structures of RA (above) and IAA (below) fractions. These structures were optimized on the basis of the data given in Table 2.

SARA method led to an abundance of polar species $\left(\mathrm{O}_{x} \mathrm{~S}_{y}\right)$ being eluted with acetone. The high relative abundance of $\mathrm{O}_{x} S_{y}$ in IAA (acetone eluted) may result from a strong interaction between the $\mathrm{O}_{x} \mathrm{~S}_{y}$ heteroatom group and the double bonded oxygen in acetone. Figure $\mathrm{S} 11$ confirms that the $\mathrm{O}_{x} \mathrm{~S}_{y}$ heteroatom group of IAA extracted by acetone is of low aromaticity (AW average $\mathrm{DBE}=9$ ).

A second elution with Heptol 1:1 led to poor extraction of both RA and IAA, with the mass yield for RA equal to $15.2 \pm$ $1.7 \%$ and negligible mass recovered for IAA, suggesting that both fractions, in particular IAA, are enriched with polar compounds. The high AW average DBE of RA extracted by Heptol 1:1 (Figure 6) confirms that the nonpolar species are highly aromatic. Moreover, a third elution with Tol/THF/MeOH $2: 2: 1$ led to a significant extraction of IAA $(77.8 \pm 6.5 \%)$, confirming the high polarity of the multi-core structures in IAA. A very high abundance of protonated $\mathrm{O}_{x} \mathrm{~S}_{y}$ species was found in IAA (Figure 3), with these highly polar species exhibiting lower DBE values than those of the radical ions. Further analysis of the extrography fractions by heteroatom groups is provided in Figures S12-S15.

Model Structures. Using the (+) APPI FT-ICR MS analysis, previous characterization data of RA and IAA, ${ }^{21,23,25}$ and other reported asphaltene characterization data, model structures for RA and IAA motifs are proposed. This is a similar approach to the development of the surrogate model structures proposed by Jameel et al., ${ }^{62}$ following extensive characterization of heavy fuel oils. ${ }^{63}$ However, rather than determining a "typical" structure for RA and IAA using only the abundance weighted average values $\left(M_{w}\right.$, carbon number, DBE), where differences were found to be negligible, five model structures for each asphaltene fraction were developed to highlight the apparent differences between the two fractions. Table 2 summarizes the parameters used to construct the model asphaltene motifs, emphasizing the abundant heteroatom groups, modal values of carbon numbers and DBEs, and size reduction by IRMPD as calculated using modal values of carbon number and DBE. The relative abundance of the heteroatom groups should be used in model structure simulations to scale the heteroatom contents to correspond to bulk fraction measurements. With scaling applied to the model structures, the $\mathrm{H} / \mathrm{C}, \mathrm{N} / \mathrm{C}, \mathrm{O} / \mathrm{C}$, and $\mathrm{S} / \mathrm{C}$ ratios of RA are $1.20,0.005,0.01$, and 0.02 , respectively, compared to $1.16,0.003,0.06$, and 0.04 for IAA. This compares to ratios of $1.04,0.02,0.06$, and 0.02 (RA) and 1.21, 0.03, 0.09, and 0.04 (IAA) from bulk elemental analysis, ${ }^{23}$ which means that the heteroatom content is slightly underestimated in the proposed model structures after scaling by the relative abundance as determined by FT-ICR MS.

The fragmentation data $(m / z 650$, Figure 5$)$ confirmed IAA to be predominantly multi-core, while RA is a mixture of singlecore and multi-core motifs. For the HC class (Figure 7), the large reduction in both carbon number and DBE (by IRMPD) for both RA and IAA indicated a predominance of multi-core structures, with the molecular size taken from the modal value of carbon number. It should be noted that the predominance of multi-core structures is likely over exaggerated as fragments containing no heteroatoms readily cleave from heteroatomcontaining groups. The $\mathrm{H} / \mathrm{C}$ mass ratios, carbon number, and $\mathrm{DBE}$ as well as heteroatom content of the proposed molecular structures in Figure 7 correspond to the FT-ICR MS data in Figures 1 and 2 and Table 2 (a consistent approach for structure determination was followed for all heteroatom groups).

The $\mathrm{O}_{x} \mathrm{~S}_{y}$ groups were found to be abundant in IAA, principally the $\mathrm{O}_{3} \mathrm{~S}_{3}$ heteroatom class (Figure 1), with these heteroatom groups mostly associated with multi-core motifs (Figure $\mathrm{S} 15)$. For IAA, the $\mathrm{O}$-containing species $\left(\mathrm{O}_{x}, \mathrm{O}_{x} \mathrm{~S}_{y}\right.$, and $\mathrm{N}_{x} \mathrm{O}_{y}$ ) accounted for $60 \%$ of the fraction by abundance (Figure S8), while their presence in RA was substantially lower (30\%) and mostly associated with single-core motifs. The remarkably low DBE values for $\mathrm{O}_{x}$ and $\mathrm{O}_{x} \mathrm{~S}_{y}$ groups in IAA (Figure 2) suggests the heteroatoms to be located in alkyl-chains rather than the aromatic cores. This has been previously suggested as likely or even typical, such as the presence of phenolic groups. ${ }^{67,68}$ Ren et al. ${ }^{31}$ confirmed the $\mathrm{O}_{1} S_{1}$ class species to be sulfoxides, but an abundance of higher-order $\mathrm{O}_{x} \mathrm{~S}_{y}$-containing species indicates the likely presence other sulfur-containing species such as sulfone. ${ }^{26,27,66}$ On the basis of previous findings, the $\mathrm{O}_{x}$ groups are most likely to be carbonyl functional groups. ${ }^{25}$

For the $S_{x}$ group, the DBEs and carbon numbers for RA and IAA were almost equivalent and showed a similar high degree of fragmentation, indicating a prevalence of multi-core structures. While the relative abundance of $S_{x}$ was found to be greater in RA than IAA, this is likely attributed to the greater association of $S$ and $\mathrm{O}$ in IAA (abundance of $\mathrm{O}_{x} \mathrm{~S}_{y}$ groups). From Figure 1, it is likely that $S_{x}$ exits as one heteroatom, which is most probably thiophene and sulfide. ${ }^{25,26,66}$

For the $\mathrm{N}$-containing species the relative abundance of $\mathrm{N}_{x}$ and $\mathrm{N}_{x} \mathrm{O}_{y}$ heteroatom groups were $11 \%$ and $5 \%$ for RA and 5\% and $8 \%$ for IAA. Table 2 shows a small reduction in the carbon number and $\mathrm{DBE}$ of the $\mathrm{N}_{x} \mathrm{O}_{y}$ group (IAA) following fragmentation, confirming its association to a large, single aromatic core with few (small) aliphatic side chains. As no $\mathrm{N}_{x}$ 
precursor ions were detected for RA, it was not possible to ascertain the degree of fragmentation.

It should be emphasized that these model structures for RA and IAA have been proposed to reflect key differences between the two fractions and are not intended to diminish the complexity of characterizing asphaltenes. Its purpose is to provide new insights regarding the molecular structure and chemistry based on abundant properties, which likely govern both bulk and interfacial behaviors of asphaltenes.

\section{CONCLUSIONS}

This study follows a methodology reported in a series of papers characterizing the physicochemical properties of interfacially active asphaltenes (IAA) and remaining asphaltenes (RA) that are extracted using the E-SARA method. Earlier studies have revealed differences in elemental composition, $M_{w}$, and nanoaggregate size and shape, yet differences in the molecular structure and chemistry of these two asphaltene fractions remained to be explored. Asphaltenes were extracted from a South American heavy crude oil and further separated into RA and IAA by the E-SARA method, with IAA accounting for $\sim 1.5$ wt $\%$ of the whole asphaltene fraction. Using positive-ion atmospheric pressure photoionization 9.4 T Fourier transform ion cyclotron resonance mass spectroscopy ((+)APPI 9.4 T FTICR MS) and infrared multiphoton dissociation (IRMPD), the discriminating properties between the two asphaltene fractions are

(1) IAA is enriched in $\mathrm{O}_{x}$ and $\mathrm{O}_{x} S_{y}$ groups with an abundance of higher-order oxygen-containing species. RA is enriched in single heteroatom classes, $\mathrm{O}_{1}, \mathrm{~S}_{1}$, and $\mathrm{N}_{1}$.

(2) For all heteroatom groups except $\mathrm{N}_{x} \mathrm{O}_{y}$, the $\mathrm{H} / \mathrm{C}$ mass ratio of IAA was higher than that of RA, confirming the less aromatic nature of IAA.

(3) Fragmentation of precursor ions $(m / z 650)$ of RA and IAA revealed a mixture of single-core and multi-core motifs in RA and an abundance of multi-core structures in IAA.

(4) IAA compounds preferentially ionize via protonation, which correlates with a higher tendency to hydrogenbond in solution.

(5) $\mathrm{N}$ - and $\mathrm{O}$-containing species are most likely found within aromatic cores and aliphatic side chains, respectively.

(6) The molecular structure of IAA is "atypical" for asphaltenes as a result of their functionality and polarity rather than high aromaticity.

Critically, this study revealed that the asphaltene fraction most likely responsible for emulsion stabilization and fouling problems does not entirely conform to the molecular description of an asphaltene. This new insight into the molecular structure and chemistry of IAA will help design appropriate chemistries to mitigate asphaltene-related problems, the success of which is strongly dependent on the cooperative interaction between the additives and asphaltene molecules/nanoaggregates.

\section{ASSOCIATED CONTENT}

\section{SI Supporting Information}

The Supporting Information is available free of charge at https://pubs.acs.org/doi/10.1021/acs.energyfuels.0c02752.

Figures of iso-abundance plots, relative abundances of the heteroatom groups, and mass spectra (PDF)

\section{AUTHOR INFORMATION}

\section{Corresponding Author}

David Harbottle - School of Chemical and Process Engineering, University of Leeds, Leeds LS2 9JT, United Kingdom; (1) orcid.org/0000-0002-0169-517X; Phone: +44(0)113343 4154; Email: d.harbottle@leeds.ac.uk

\section{Authors}

Dewi A. Ballard - School of Chemical and Process Engineering, University of Leeds, Leeds LS2 9JT, United Kingdom; (1) orcid.org/0000-0002-5506-199X

Martha L. Chacón-Patiño - National High Magnetic Field Laboratory, Florida State University, Tallahassee, Florida 32306, United States; (1) orcid.org/0000-0002-7273-5343

Peiqi Qiao - Department of Chemical and Materials Engineering, University of Alberta, Edmonton AB T6G 2R3, Canada

Kevin J. Roberts - School of Chemical and Process Engineering, University of Leeds, Leeds LS2 9JT, United Kingdom; (i) orcid.org/0000-0002-1070-7435

Robert Rae - Infineum UK Ltd., Abingdon, Oxfordshire OX13 6BB, United Kingdom

Peter J. Dowding - Infineum UK Ltd., Abingdon, Oxfordshire OX13 6BB, United Kingdom

Zhenghe Xu - Department of Chemical and Materials Engineering, University of Alberta, Edmonton AB T6G 2R3, Canada; Department of Materials Science and Engineering, Southern University of Science and Technology, Shenzhen 518055, China; (1) orcid.org/0000-0001-8118-1920

Complete contact information is available at:

https://pubs.acs.org/10.1021/acs.energyfuels.0c02752

\section{Notes}

The authors declare no competing financial interest. Article metadata is available under the Creative Commons Attribution license (CC-BY). ${ }^{69}$

\section{ACKNOWLEDGMENTS}

This research was completed at the EPSRC Centre for Doctoral Training in Complex Particulate Products and Process (EP/ L015285/1) in collaboration with Infineum UK Ltd., who we greatly acknowledge for their support. D.H. acknowledges funding from the Royal Academy of Engineering IndustryAcademia Partnership Program (IAPP1/100150). Z.X. acknowledges funding from the Natural Sciences and Engineering Research Council (NSERC) - Industrial Research Chair (IRC) Program in Oil Sands Engineering. A portion of this work was performed at the National High Magnetic Field Laboratory, which is supported by the National Science Foundation Cooperative Agreement No. DMR-1644779 and the State of Florida. The authors would like to thank Donald Smith for help with data calibration and Yuri E. Corilo for the PetroOrg software.

\section{ABBREVIATIONS}

APPI, atmospheric pressure photoionization; AW, abundance weighted; E-SARA, extended-SARA; FT-ICR MS, Fourier transform ion cyclotron resonance mass spectrometry; IAA, interfacially active asphaltenes; IRMPD, infrared multi-photon dissociation; NALDI-MS, nanostructure-assisted laser desorption mass spectroscopy; RA, remaining asphaltenes; SWIFT, stored waveform inverse Fourier transform; WA, whole asphaltenes 


\section{REFERENCES}

(1) McKenna, A. M.; Chacón-Patiño, M. L.; Weisbrod, C. R.; Blakney, G. T.; Rodgers, R. P. Molecular-Level Characterization of Asphaltenes Isolated from Distillation Cuts. Energy Fuels 2019, 33 (3), 2018-2029.

(2) Gray, M. R.; Tykwinski, R. R.; Stryker, J. M.; Tan, X. Supramolecular Assembly Model for Aggregation of Petroleum Asphaltenes. Energy Fuels 2011, 25 (7), 3125-3134.

(3) Mullins, O. C. The Asphaltenes. Annu. Rev. Anal. Chem. 2011, 4 (1), 393-418.

(4) Schuler, B.; Zhang, Y.; Liu, F.; Pomerantz, A. E.; Andrews, A. B.; Gross, L.; Pauchard, V.; Banerjee, S.; Mullins, O. C. Overview of Asphaltene Nanostructures and Thermodynamic Applications. Energy Fuels, in press, 2020.

(5) Chacón-Patiño, M. L.; Rowland, S. M.; Rodgers, R. P. Advances in Asphaltene Petroleomics. Part 1: Asphaltenes Are Composed of Abundant Island and Archipelago Structural Motifs. Energy Fuels 2017, 31 (12), 13509-13518.

(6) Qiao, P.; Harbottle, D.; Tchoukov, P.; Masliyah, J.; Sjoblom, J.; Liu, Q.; Xu, Z. Fractionation of Asphaltenes in Understanding Their Role in Petroleum Emulsion Stability and Fouling. Energy Fuels 2017, 31 (4), 3330-3337.

(7) Mullins, O.; Sheu, E.; Hammami, A.; Marshall, A. Asphaltenes, Heavy Oils, and Petroleomics; Springer New York: New York, NY, 2007.

(8) Spiecker, P. M.; Gawrys, K. L.; Kilpatrick, P. K. Aggregation and solubility behavior of asphaltenes and their subfractions. J. Colloid Interface Sci. 2003, 267 (1), 178-93.

(9) Wattana, P.; Fogler, H. S.; Yen, A.; Carmen Garcìa, M. D.; Carbognani, L. Characterization of Polarity-Based Asphaltene Subfractions. Energy Fuels 2005, 19 (1), 101-110.

(10) Pinto, F. E.; Barros, E. V.; Tose, L. V.; Souza, L. M.; Terra, L. A.; Poppi, R. J.; Vaz, B. G.; Vasconcelos, G.; Subramanian, S.; Simon, S.; Sjöblom, J.; Romão, W. Fractionation of asphaltenes in n-hexane and on adsorption onto $\mathrm{CaCO} 3$ and characterization by ESI(+)FT-ICR MS: Part I. Fuel 2017, 210, 790-802.

(11) Kilpatrick, P. K. Water-in-Crude Oil Emulsion Stabilization: Review and Unanswered Questions. Energy Fuels 2012, 26 (7), 40174026.

(12) Spiecker, P. M.; Kilpatrick, P. K. Interfacial Rheology of Petroleum Asphaltenes at the Oil-Water Interface. Langmuir 2004, 20 (10), 4022-4032.

(13) Verruto, V. J.; Le, R. K.; Kilpatrick, P. K. Adsorption and Molecular Rearrangement of Amphoteric Species at Oil-Water Interfaces. J. Phys. Chem. B 2009, 113 (42), 13788-13799.

(14) Wu, X. Investigating the Stability Mechanism of Water-inDiluted Bitumen Emulsions through Isolation and Characterization of the Stabilizing Materials at the Interface. Energy Fuels 2003, 17 (1), 179-190.

(15) Stanford, L. A.; Rodgers, R. P.; Marshall, A. G.; Czarnecki, J.; Wu, X. A.; Taylor, S. Detailed Elemental Compositions of Emulsion Interfacial Material versus Parent Oil for Nine Geographically Distinct Light, Medium, and Heavy Crude Oils, Detected by Negative- and Positive-Ion Electrospray Ionization Fourier Transform Ion Cyclotron Resonance Mass Spectrometry. Energy Fuels 2007, 21 (2), 973-981.

(16) Stanford, L. A.; Rodgers, R. P.; Marshall, A. G.; Czarnecki, J.; Wu, X. A. Compositional Characterization of Bitumen/Water Emulsion Films by Negative- and Positive-Ion Electrospray Ionization and Field Desorption/Ionization Fourier Transform Ion Cyclotron Resonance Mass Spectrometry. Energy Fuels 2007, 21 (2), 963-972.

(17) Czarnecki, J. Stabilization of Water in Crude Oil Emulsions. Part 2. Energy Fuels 2009, 23 (3), 1253-1257.

(18) Subramanian, S.; Simon, S.; Gao, B.; Sjöblom, J. Asphaltene fractionation based on adsorption onto calcium carbonate: Part 1 . Characterization of sub-fractions and QCM-D measurements. Colloids Surf., A 2016, 495 (Supplement C), 136-148.

(19) Subramanian, S.; Sørland, G. H.; Simon, S.; Xu, Z.; Sjöblom, J. Asphaltene fractionation based on adsorption onto calcium carbonate: Part 2. Self-association and aggregation properties. Colloids Surf., A 2017, 514, 79-90.
(20) Rogel, E.; Ovalles, C.; Moir, M. Asphaltene Chemical Characterization as a Function of Solubility: Effects on Stability and Aggregation. Energy Fuels 2012, 26 (5), 2655-2662.

(21) Yang, F.; Tchoukov, P.; Dettman, H.; Teklebrhan, R. B.; Liu, L.; Dabros, T.; Czarnecki, J.; Masliyah, J.; Xu, Z. Asphaltene Subfractions Responsible for Stabilizing Water-in-Crude Oil Emulsions. Part 2: Molecular Representations and Molecular Dynamics Simulations. Energy Fuels 2015, 29 (8), 4783-4794.

(22) Yang, F.; Tchoukov, P.; Pensini, E.; Dabros, T.; Czarnecki, J.; Masliyah, J.; Xu, Z. Asphaltene Subfractions Responsible for Stabilizing Water-in-Crude Oil Emulsions. Part 1: Interfacial Behaviors. Energy Fuels 2014, 28 (11), 6897-6904.

(23) Ballard, D. A.; Qiao, P.; Cattoz, B.; Dowding, P. J.; Prevost, S.; Alshamsi, M.; Charpentier, T.; Roberts, K. J.; Xu, Z.; Harbottle, D. Aggregation Behavior of E-SARA Asphaltene Fractions Studied by Small-Angle Neutron Scattering. Energy Fuels 2020, 34 (6), 68946903.

(24) Qiao, P.; Harbottle, D.; Li, Z.; Tang, Y.; Xu, Z. Interactions of Asphaltene Subfractions in Organic Media of Varying Aromaticity. Energy Fuels 2018, 32 (10), 10478-10485.

(25) Qiao, P.; Harbottle, D.; Tchoukov, P.; Wang, X.; Xu, Z. Asphaltene Subfractions Responsible for Stabilizing Water-in-Crude Oil Emulsions. Part 3. Effect of Solvent Aromaticity. Energy Fuels 2017, 31 (9), 9179-9187.

(26) Mitra-Kirtley, S.; Mullins, O. C.; Ralston, C. Y.; Sellis, D.; Pareis, C. Determination of Sulfur Species in Asphaltene, Resin, and Oil Fractions of Crude Oils. Appl. Spectrosc. 1998, 52 (12), 1522-1525.

(27) Waldo, G. S.; Mullins, O. C.; Penner-Hahn, J. E.; Cramer, S. P. Determination of the chemical environment of sulphur in petroleum asphaltenes by X-ray absorption spectroscopy. Fuel 1992, 71 (1), 5357.

(28) Wiltfong, R.; Mitra-Kirtley, S.; Mullins, O. C.; Andrews, B.; Fujisawa, G.; Larsen, J. W. Sulfur Speciation in Different Kerogens by XANES Spectroscopy. Energy Fuels 2005, 19 (5), 1971-1976.

(29) Sinninghe Damste, J. S.; De Leeuw, J. W. Analysis, structure and geochemical significance of organically-bound sulphur in the geosphere: State of the art and future research. Org. Geochem. 1990, 16 (4), 1077-1101.

(30) Payzant, J. D.; Montgomery, D. S.; Strausz, O. P. Novel terpenoid sulfoxides and sulfides in petroleum. Tetrahedron Lett. 1983, 24 (7), 651-654.

(31) Ren, L.; Wu, J.; Qian, Q.; Liu, X.; Meng, X.; Zhang, Y.; Shi, Q. Separation and Characterization of Sulfoxides in Crude Oils. Energy Fuels 2019, 33 (2), 796-804.

(32) Chacón-Patiño, M. L.; Rowland, S. M.; Rodgers, R. P. Advances in Asphaltene Petroleomics. Part 2: Selective Separation Method That Reveals Fractions Enriched in Island and Archipelago Structural Motifs by Mass Spectrometry. Energy Fuels 2018, 32 (1), 314-328.

(33) Chacón-Patiño, M. L.; Rowland, S. M.; Rodgers, R. P. Advances in Asphaltene Petroleomics. Part 3. Dominance of Island or Archipelago Structural Motif Is Sample Dependent. Energy Fuels 2018, 32 (9), 9106-9120.

(34) Chacón-Patiño, M. L.; Smith, D. F.; Hendrickson, C. L.; Marshall, A. G.; Rodgers, R. P. Advances in Asphaltene Petroleomics. Part 4. Compositional Trends of Solubility Subfractions Reveal that Polyfunctional Oxygen-Containing Compounds Drive Asphaltene Chemistry. Energy Fuels 2020, 34 (3), 3013-3030.

(35) Strausz, O. P.; Torres, M.; Lown, E. M.; Safarik, I.; Murgich, J. Equipartitioning of Precipitant Solubles between the Solution Phase and Precipitated Asphaltene in the Precipitation of Asphaltene. Energy Fuels 2006, 20 (5), 2013-2021.

(36) Chacón-Patiño, M. L.; Vesga-Martínez, S. J.; Blanco-Tirado, C.; Orrego-Ruiz, J. A.; Gómez-Escudero, A.; Combariza, M. Y. Exploring Occluded Compounds and Their Interactions with Asphaltene Networks Using High-Resolution Mass Spectrometry. Energy Fuels 2016, 30 (6), 4550-4561.

(37) Juyal, P.; McKenna, A. M.; Fan, T.; Cao, T.; Rueda-Velásquez, R. I.; Fitzsimmons, J. E.; Yen, A.; Rodgers, R. P.; Wang, J.; Buckley, J. S.; 
Gray, M. R.; Allenson, S. J.; Creek, J. Joint Industrial Case Study for Asphaltene Deposition. Energy Fuels 2013, 27 (4), 1899-1908.

(38) Chacón-Patiño, M. L.; Blanco-Tirado, C.; Orrego-Ruiz, J. A.; Gómez-Escudero, A.; Combariza, M. Y. High Resolution Mass Spectrometric View of Asphaltene-SiO2 Interactions. Energy Fuels 2015, 29 (3), 1323-1331.

(39) Loegel, T. N.; Danielson, N. D.; Borton, D. J.; Hurt, M. R.; Kenttämaa, H. I. Separation of Asphaltenes by Reversed-Phase Liquid Chromatography with Fraction Characterization. Energy Fuels 2012, 26 (5), 2850-2857.

(40) Tapio, L.; Subramanian, S.; Simon, S.; Sjöblom, J. Solvent desorption of asphaltenes from solid surfaces. J. Dispersion Sci. Technol. 2017, 38 (3), 355-360.

(41) Robb, D. B.; Covey, T. R.; Bruins, A. P. Atmospheric Pressure Photoionization: An Ionization Method for Liquid ChromatographyMass Spectrometry. Anal. Chem. 2000, 72 (15), 3653-3659.

(42) Kaiser, N. K.; Quinn, J. P.; Blakney, G. T.; Hendrickson, C. L.; Marshall, A. G. A Novel 9.4 T FTICR Mass Spectrometer with Improved Sensitivity, Mass Resolution, and Mass Range. J. Am. Soc. Mass Spectrom. 2011, 22 (8), 1343-1351.

(43) Beu, S. C.; Blakney, G. T.; Quinn, J. P.; Hendrickson, C. L.; Marshall, A. G. Broadband Phase Correction of FT-ICR Mass Spectra via Simultaneous Excitation and Detection. Anal. Chem. 2004, 76 (19), $5756-5761$.

(44) Blakney, G. T.; Hendrickson, C. L.; Marshall, A. G. Predator data station: A fast data acquisition system for advanced FT-ICR MS experiments. Int. J. Mass Spectrom. 2011, 306 (2), 246-252.

(45) Corilo, Y. E. PetroOrg. N-18.3; Florida State University, Omics LLC: Tallahassee, FL, 2014.

(46) Rytting, B. M.; Harper, M. R.; Edmond, K. V.; Merchant, S.; Zhang, Y.; Kilpatrick, P. K. Interfacial Phenomena of Purified Petroporphyrins and Their Impact on Asphaltene Interfacial Film Formation. Energy Fuels 2020, 34 (5), 5444-5456.

(47) Rytting, B. M.; Harper, M. R.; Edmond, K. V.; Zhang, Y.; Kilpatrick, P. K. High-Purity Vanadyl Petroporphyrins: Their Aggregation and Effect on the Aggregation of Asphaltenes. Energy Fuels 2020, 34 (1), 164-178.

(48) Schulze, M.; Lechner, M. P.; Stryker, J. M.; Tykwinski, R. R. Aggregation of asphaltene model compounds using a porphyrin tethered to a carboxylic acid. Org. Biomol. Chem. 2015, 13 (25), 6984-6991.

(49) Hockaday, W. C.; Purcell, J. M.; Marshall, A. G.; Baldock, J. A.; Hatcher, P. G. Electrospray and photoionization mass spectrometry for the characterization of organic matter in natural waters: a qualitative assessment. Limnol. Oceanogr.: Methods 2009, 7 (1), 81-95.

(50) Purcell, J. M.; Hendrickson, C. L.; Rodgers, R. P.; Marshall, A. G. Atmospheric pressure photoionization proton transfer for complex organic mixtures investigated by fourier transform ion cyclotron resonance mass spectrometry. J. Am. Soc. Mass Spectrom. 2007, 18 (9), $1682-1689$.

(51) Hertzog, J.; Carré, V.; Le Brech, Y.; Mackay, C. L.; Dufour, A.; Mašek, O.; Aubriet, F. Combination of electrospray ionization, atmospheric pressure photoionization and laser desorption ionization Fourier transform ion cyclotronic resonance mass spectrometry for the investigation of complex mixtures - Application to the petroleomic analysis of bio-oils. Anal. Chim. Acta 2017, 969, 26-34.

(52) McKenna, A. M.; Marshall, A. G.; Rodgers, R. P. Heavy Petroleum Composition. 4. Asphaltene Compositional Space. Energy Fuels 2013, 27 (3), 1257-1267.

(53) Boduszynski, M. M. Composition of heavy petroleums. 1. Molecular weight, hydrogen deficiency, and heteroatom concentration as a function of atmospheric equivalent boiling point up to 1400.degree.F (760.degree.C). Energy Fuels 1987, 1 (1), 2-11.

(54) Boduszynski, M. M.; Altgelt, K. H. Composition of heavy petroleums. 4. Significance of the extended atmospheric equivalent boiling point (AEBP) scale. Energy Fuels 1992, 6 (1), 72-76.

(55) McKenna, A. M.; Blakney, G. T.; Xian, F.; Glaser, P. B.; Rodgers, R. P.; Marshall, A. G. Heavy Petroleum Composition. 2. Progression of the Boduszynski Model to the Limit of Distillation by Ultrahigh-
Resolution FT-ICR Mass Spectrometry. Energy Fuels 2010, 24 (5), 2939-2946.

(56) Chacón-Patiño, M. L.; Rowland, S. M.; Rodgers, R. P. The Compositional and Structural Continuum of Petroleum from Light Distillates to Asphaltenes: The Boduszynski Continuum Theory As Revealedby FT-ICR Mass Spectrometry. The Boduszynski Continuum: Contributions to the Understanding of the Molecular Composition of Petroleum; American Chemical Society: Washington, DC, 2018; Vol. 1282, pp 113-171.

(57) Nyadong, L.; Lai, J.; Thompsen, C.; LaFrancois, C. J.; Cai, X.; Song, C.; Wang, J.; Wang, W. High-Field Orbitrap Mass Spectrometry and Tandem Mass Spectrometry for Molecular Characterization of Asphaltenes. Energy Fuels 2018, 32 (1), 294-305.

(58) Dong, X.; Zhang, Y.; Milton, J.; Yerabolu, R.; Easterling, L.; Kenttämaa, H. I. Investigation of the relative abundances of single-core and multicore compounds in asphaltenes by using high-resolution insource collision-activated dissociation and medium-energy collisionactivated dissociation mass spectrometry with statistical considerations. Fuel 2019, 246, 126-132.

(59) Podgorski, D. C.; Corilo, Y. E.; Nyadong, L.; Lobodin, V. V.; Bythell, B. J.; Robbins, W. K.; McKenna, A. M.; Marshall, A. G.; Rodgers, R. P. Heavy Petroleum Composition. 5. Compositional and Structural Continuum of Petroleum Revealed. Energy Fuels 2013, 27 (3), 1268-1276.

(60) Murgich, J. Molecular Simulation and the Aggregation of the Heavy Fractions in Crude Oils. Mol. Simul. 2003, 29 (6-7), 451-461.

(61) Chung, S.-H.; Violi, A. Peri-condensed aromatics with aliphatic chains as key intermediates for the nucleation of aromatic hydrocarbons. Proc. Combust. Inst. 2011, 33 (1), 693-700.

(62) Abdul Jameel, A. G.; Khateeb, A.; Elbaz, A. M.; Emwas, A.-H.; Zhang, W.; Roberts, W. L.; Sarathy, S. M. Characterization of deasphalted heavy fuel oil using APPI (+) FT-ICR mass spectrometry and NMR spectroscopy. Fuel 2019, 253, 950-963.

(63) Elbaz, A. M.; Gani, A.; Hourani, N.; Emwas, A.-H.; Sarathy, S. M.; Roberts, W. L. TG/DTG, FT-ICR Mass Spectrometry, and NMR Spectroscopy Study of Heavy Fuel Oil. Energy Fuels 2015, 29 (12), $7825-7835$

(64) Mitra-Kirtley, S.; Mullins, O. C.; Van Elp, J.; George, S. J.; Chen, J.; Cramer, S. P. Determination of the nitrogen chemical structures in petroleum asphaltenes using XANES spectroscopy. J. Am. Chem. Soc. 1993, $115(1), 252-258$.

(65) Simon, S.; Nenningsland, A. L.; Herschbach, E.; Sjöblom, J. Extraction of Basic Components from Petroleum Crude Oil. Energy Fuels 2010, 24 (2), 1043-1050.

(66) Zhang, L.-L.; Wang, C.-L.; Zhao, Y.-S.; Yang, G.-H.; Su, M.; Yang, C.-H. Speciation and quantification of sulfur compounds in petroleum asphaltenes by derivative XANES spectra. Journal of Fuel Chemistry and Technology 2013, 41 (11), 1328-1335.

(67) Nahid Siddiqui, M. Infrared Study of Hydrogen Bond Types in Asphaltenes. Pet. Sci. Technol. 2003, 21 (9-10), 1601-1615.

(68) Mullins, O. C. The Modified Yen Model. Energy Fuels 2010, 24 (4), 2179-2207.

(69) Ballard, D. A.; Chacón-Patiño, M. L.; Qiao, P.; Roberts, K. J.; Rae, R.; Dowding, P. J.; Xu, Z.; Harbottle, D. Data associated with 'Molecular Characterization of Strongly and Weakly Interfacially Active Asphaltenes by High-Resolution Mass Spectrometry', University of Leeds, 2020. [Dataset] DOI: 10.5518/883. 